\title{
Klinisch-numerische Forschung in der britischen Geburtshilfe 1750-1820*
}

\author{
Von Ulrich Tröhler
}

I

Eine Anzahl an Allgemeinspitälern tätiger britischer Ärzte und Chirurgen begann in der zweiten Hälfte des 18. Jahrhunderts mit der zahlenmäßigen Analyse eigener Beobachtungsreihen zur Erarbeitung einer sicheren Grundlage von Krankheitsbeschreibung und Therapie ${ }^{1,2,3}$. Die Tatsache, daß in Großbritannien zwischen 1745 und 1800 mindestens dreizehn spezielle Gebärspitäler eröffnet wurden und daß es dort (neben der ersten europäischen Professur in Edinburgh) in William Smellie (1697-1763), William Hunter (1718-1783) und Charles White (1728-1813) einige der findigsten Geburtshelfer jener Zeit gab ${ }^{4}$, die althergebrachter Theorie eigene Beobachtung zur Seite stellten ${ }^{5}$, legt uns die Suche nach numerischer Forschung auch in der Geburtshilfe nahe. In dieser Richtung sollen in der vorliegenden Arbeit zwei Fragenkreise untersucht werden: das Kindbettfieber und die Indikationen zu einigen operativen Eingriffen.

\section{II}

Die «Fieber» gehörten damals zu den meistdiskutierten medizinischen Fragen ${ }^{6}$. Sie schlossen das Kindbettfieber ein, wobei Beiträge britischer Autoren durchaus im Vordergrund standen ${ }^{7}$. Diese Komplikation trat mit der häufiger werdenden aktiven Geburtshilfe und mit der Eröffnung eigentlicher Gebärkliniken in epidemischem Ausmaß auf ${ }^{8}$. Dies und die angewandte Therapie ist mit ein Grund für die Verurteilung von Ärzten und Spitälern des 18. Jahrhunderts durch einige heutige Historiker ${ }^{9}$.

Tatsächlich behandelte man das Puerperalfieber bei den ersten Ausbrüchen in London um 1760 in gänzlich traditioneller Weise mit kräftigem Aderlaß. Thomas Denman (1733-1815), John Leake (1729-1792) und Nathaniel Hulme (1732-1807) empfahlen den Blutentzug auf rein theoreti-

${ }^{*}$ Herrn Prof. Dr. med. H. Buess zum 70. Geburtstag gewidmet. 
scher Basis und ohne Erfolgskontrolle ${ }^{10}$. Bedenkt man, daß sie alle einem Gebärspital vorstanden ${ }^{11}$, so scheinen die heutigen Vorwürfe auf den ersten Blick nicht ungerechtfertigt. Man übersieht dabei allerdings, daß ohne Zuwendung zur «unbefangenen» Auswertung eigener Beobachtungen eine Abkehr von galenischen Auffassungen auch in anderen Sparten nicht denkbar war. Beim Kindbettfieber, das plötzlich an einem Ort gehäuft auftrat, mag nun das unmittelbare Erkennen der trotz «bewährter» Behandlung hohen Mortalität einen Anstoß zur Statistik gegeben haben.

Der oft zitierte Bericht über eine Epidemie von 1746 im Hôtel-Dieu in Paris ${ }^{12}$, der ersten im 18. Jahrhundert ${ }^{13}$, die mit dem Tod aller 27 Erkrankten endete, wird einige aufgerüttelt haben ${ }^{14}$. Kurz danach beobachtete William Hunter am British Lying-in Hospital, London, innerhalb dreier Monate 23 Fälle (mit höchstens zwei Heilungen), wie er seinen Studenten berichtete ${ }^{15}$. 1793 übersah John Clarke (1761-1815) vom General Lying-in Hospital, London, bereits die proportionale Morbidität und Mortalität bei sechs Ausbrüchen in England und Schottland zwischen 1760 und $1773^{16}$.

Auch der Aderlasser Leake führte Buch über alle Patientinnen seines 1767 eröffneten New Westminster Lying-in Hospital. Bereits 1769/70 wurde es vom Kindbettfieber heimgesucht. Im Winterhalbjahr verlor er von 64 Entbundenen 14 der 19 Erkrankten ${ }^{17}$. Fielen ihm die Schuppen von den Augen? Vorderhand nicht, denn die Sterblichkeit in seinem Spital war geringer als am British Lying-in Hospital des als konservativ bekannten Hunter! Zudem schloß Leake aus dem Vergleich der Morbidität und Mortalität an seinem Spital mit derjenigen der Londoner Sterbetafeln, den Bills of mortality, daß die Ätiologie der Epidemie in der «bösartigen Konstellation der Luft» liegen müsse - also unbeeinflußbar sei. Die numerischen Bestätigungen vorherrschender Meinungen bestärkten ihn in seiner unbedingten Empfehlung des Aderlasses ${ }^{18}$. Dies war übrigens ganz im Sinne Smellies. Dieser gab in seinem immer noch aufgelegten Lehrbuch dieselbe Therapie an, obschon er offensichtlich keine Epidemie erlebt hatte, als er es zwischen 1746 und 1752 verfaßte: In seinem Band illustrativer Fälle fehlt das Kindbettfieber überhaupt ${ }^{19}$.

Leake bedauerte allerdings in seinen Practical observations on the child-bed fever (1772), daß die Bills of mortality «immer noch in einer Weise geführt werden, die ihrem ursprünglichen Verwendungszweck zuwiderläuft und alle darauf beruhenden Berechnungen vage und unbestimmt werden läßt». ${ }^{20} \mathrm{Er}$ war nicht der einzige englische Arzt, der sich nach 1750 damit auseinandersetzte. Mehr und mehr schätzten Mediziner im Lauf des 18. Jahrhunderts 
genaue Angaben über die Sterblichkeit für den Nachweis, daß Reformen und präventivmedizinische Maßnahmen im Gesundheitswesen der wachsenden Städte notwendig seien ${ }^{21}$. Als Gründer und «Promotor» seines Spitals ${ }^{22}$ war Leake wohl an solchem Zahlenmaterial interessiert. Und aus dieser Sicht war auch der zweimal aufgelegte Nachdruck der Jahrgänge seit 1601 der Bills of mortality, finanziert vom Arzt William Heberden (1710-1801), in den 1750 iger Jahren ${ }^{23}$ kein Zufall.

Über statistische Erhebungen für eine auf praktischen Nutzen in der Medizin auszurichtende Sterbetafel mögen 1773 in Manchester auch einige der Angaben zur Geburtshilfe durch William Hunters hervorragenden Schüler Charles White zustande gekommen sein. Ein Jahr zuvor hatte dort Whites Freund, der vielseitige Arzt Thomas Percival (1740-1804), einen Plan für eine «genauere und vollständigere Erarbeitung der Sterbelisten in Manchester» veröffentlicht und mit dessen Durchführung begonnen. Dabei verglich er die Sterblichkeit in der Stadt mit jener in der Umgebung. Das Ergebnis fiel sehr zum Nachteil der Stadt aus, obwohl das Klima gleich war ${ }^{24}$. Zweifel an Sydenhams Klima-Pathologie, die zu einer Modeströmung wurde ${ }^{25}$, waren damit gesät: White schrieb die Entstehung des Fiebers nicht der allgemeinen Wetterlage, sondern spezifisch den damals üblichen überhitzten, ungelüfteten Gebärzimmern zu, worin die Mütter zudem noch lange bettlägerig zu bleiben hatten. Deshalb hielt er gezielte Präventivmaßnahmen (Ventilation, Frühmobilisation) für geeignet, ein Fieber zu verhindern, an dem er selbst nie eine Patientin verloren hatte ${ }^{26}$.

Als Grundlage seiner hygienischen Empfehlungen diente White die allgemeine Statistik der Manchester Infirmary, wo die Mortalität seit dem Umzug in durchlüftete und saubere Räumlichkeiten von 5,5 auf 4,1 Prozent gesunken war ${ }^{27}$. Zur Behandlung machte er Angaben nach den neuesten, experimentell untermauerten pathophysiologischen Erklärungen des Fiebers ${ }^{28}$. Anstatt die Entzündung mit einem Aderlaß zu entziehen, wollte er ihr bei Anstieg des Thermometers (sic!) mit Kälte-, bei Abfall mit Wärmeapplikation entgegentreten ${ }^{29}$.

Whites Treatise erschien in seiner letzten Auflage 1791. Bis dahin widersetzten sich mehrere Autoren über Kindbettfieber der «schwächenden» venaesectio ${ }^{30}$. Sie empfahlen statt dessen im Sinne Whites die Isolation und ein Stimulans, Perubalsam, der damals auf statistischer Grundlage auch in die Typhustherapie eingeführt wurde ${ }^{31}$.

Hinderten äußere Gründe ${ }^{32}$ White daran, seine Empfehlungen zahlenmäßig zu überprüfen, wie er das für seine Amputationsmethode tat ${ }^{33}$ ? Wie 
dem auch sei, numerische Ergebnisse ließen von anderer Seite nicht lange auf sich warten: Robert Bland (1730-1816) «nützte die Vorteile seiner Stellung ... am Westminster Dispensary» aus, wie sich sein Kollege Samuel Merriman (1731-1818) ausdrückte ${ }^{34}$; denn er machte seit der Eröffnung dieser Poliklinik 1774 Aufzeichnungen über jede Patientin ${ }^{35} .1781$ veröffentlichte er die vollständigen Resultate seiner Praxis in der Londoner Royal Society. Ebendort publizierte 1786 Joseph Clarke (1758-1834) diejenigen der berühmten Dubliner Rotunda-Gebärklinik ${ }^{36}$. Diese Arbeiten zeigten den Erfolg der mit Bezug auf White ${ }^{37}$ getroffenen vorbeugenden Vorkehrungen eindrücklich. Bland registrierte nur fünf Fälle von Puerperalfieber (davon vier tödliche) bei 1897 Geburten in sieben Jahren, während Clarke 229 Todesfälle bei 19786 Entbindungen zwischen 1757 und 1784 verzeichnete. Eine spätere Statistik der Jahre 1787-1793 sollte seine Ablehnung der Aderlaßtherapie des Puerperalfiebers begründen. Trotz einer Epidemie hatte er von 10387 Patientinnen nur 68 an Leiden verloren, die damit in Zusammenhang gebracht werden konnten ${ }^{38}$. Ohne Angabe der Morbidität stellten diese Zahlen allerdings keinen direkten Erfolgsnachweis von Clarkes Therapie dar ${ }^{39}$.

Solche Angaben, wenn auch unerwarteter Art, publizierte 1795 der am Aeberdeen Dispensary wirkende Alexander Gordon (1752-1799). Seine tabellarische Darstellung der Kindbettfieberepidemie von 1789 in Aeberdeen mit den Erkrankungsdaten, den Namen der Hebammen und Ärzte jeder Patientin, mit der er die Ansteckung erstmals direkt nachwies, ist wohlbekannt ${ }^{40}$. Dagegen weiß man kaum, daß dieser ehemalige Schiffschirurg und Praktikant am Middlesex Hospital in London in seinem Treatise on the ... puerperal fever ...41 ebenso klar und zahlenmäßig die Wirksamkeit der venaesectio belegte. «Nichts kann ein stärkerer Beweis für die Richtigkeit meiner Doktrin sein als der Erfolg meiner Praxis», erklärte er in typisch empirischer Redeweise seiner Zeit ${ }^{42}$. In der Tat hatte er von 77 Krankheitsfällen nur 28 verloren, also bedeutend weniger als Leake oder gar Bland, wie er hervorhob; ja, wenn man die 27 «zu spät» (d.h. nach 24 Stunden) behandelten Patientinnen ausschloß, waren von den verbleibenden 50 nur 5 gestorben ${ }^{43}$. "The cure is severe», bemerkte er lakonisch, «but it is only short, for the patient is cured in a few days, or not at all.» ${ }^{44}$ Von Prävention hielt Gordon, abgesehen vom Händewaschen, nicht viel. Am liebsten hätte er vorbeugend zur Ader gelassen; doch wollte er dieses einzig sichere Mittel in einem eventuellen Ernstfall nicht entbehren und ließ es deshalb mit einer Purganz bewenden. 
Sieben Autopsien hatten am Anfang der Epidemie in Aberdeen das Bild einer lokalisierten Entzündung ergeben ${ }^{45}$, der man nur mit sofortigem massivem Aderlaß (20-24 Unzen, also mehr als $600 \mathrm{ml}$ pro Mal) Meister wurde, wie nun die Statistik belegte. Damit leitete Gordon bereits 1795 eine abermalige Renaissance des Aderlasses in England ein, die nach $1805^{46}$ wahllos auch andere «Fieber» erfaßte. William Hey (1772-1844) und John Armstrong (1784-1829) berichteten um 1815 begeistert aus Leeds und London zahlenmäßig über ihre neuesten Erfolge bei Kindbettfieber, besonders wenn sie diese mit Resultaten vor der Einführung der Gordonschen Therapie am gleichen Spital verglichen ${ }^{47}$. Joseph Clarke allerdings blieb in seiner Ablehnung des Aderlasses fest. Er schrieb an John Armstrong, er sehe keinen Grund, auch weiterhin auf den Aderlaß nach der Geburt zu verzichten, da in seinem Spital seit 1757 nur 875 von 84390 Patientinnen gestorben seien. Er meinte, Armstrongs sogenannte Erfolge hingen mit der ungenauen Definition des Kindbettfiebers zusammen ${ }^{48}$.

Mit dieser einsichtigen Feststellung charakterisierte Clarke den Wissensstand seiner Zeit trefflich. Trotz der Verwendung von Statistiken blieben Fieberforschung und -therapie unsicher und daher Modeströmungen ausgeliefert. Vielleicht leisteten die Zahlen diesen noch Vorschub, wie es heute noch für ähnliche Situationen zutreffen mag.

\section{III}

Statistische Daten dienten auch zur Erforschung einiger Grundfragen der Geburtshilfe. Bland und Merriman betonten, daß damit Smellies und Leakes erstmalige Schätzungen über die Inzidenz verschiedener Präsentationen, über Auftreten und Verlauf von Geburtskomplikationen und damit das Erfassen bestimmter Indikationen zum Gebrauch der Geburtszange präzisiert werden sollten ${ }^{49}$. Joseph Clarke und Robert Bland interessierten sich daneben auch für die Geschlechts-, Gewichts- und Größenverteilung der Kinder, ihre Sterblichkeit, die Häufigkeit von Mehrfachgeburten und Mißgeburten. Für diese Forschung versandte Bland 1400 Fragebogen an ehemalige Patientinnen und erhielt heute noch gültige Resultate ${ }^{50}$. Samuel Merriman (1731-1818) und Augustus Bozzi-Granville (1783-1872) in London und Robert Collins (1801-1896) in Dublin setzten diese Arbeiten fort und dehnten sie auf die Fehlgeburten aus, deren Risiko sie nach dem Alter der Mutter und dem Zeitpunkt der Schwangerschaft genau zu ermitteln trachteten ${ }^{51}$. 
Edward Rigby (1747-1821), Chirurg am Norfolk and Norwich Hospital, beendete eine jahrhundertealte Kontroverse um die Blutungen unter der Geburt. Er teilte sie nach klaren Kriterien ein in «akzidentelle» und «unabwendbare», wobei letztere dem Vorliegen einer placenta praevia entsprachen ${ }^{52}$. Wie er selbst anerkannte, gebührte ihm die Priorität für diese Unterscheidung nicht, obschon er von den vier vorher von André Levret (1703-1780) in Paris mitgeteilten Fällen nichts gewußt hatte ${ }^{53}$. Sein Verdienst lag in der klaren statistischen Untermauerung seiner Differentialdiagnose und ihrer therapeutischen Konsequenzen. Die Angaben in seinem Essay on the uterine haemorrhage beruhten 1775 bereits auf allen 36, in der vierten Auflage 1789 auf den 106 eigenen, seit 1769 registrierten, nun numerisch analysierten Fällen. Bei placenta praevia empfahl er sofortige Beendigung der Geburt durch Wendung und Extraktion, während bei akzidenteller Blutung ruhig der natürliche Verlauf abgewartet werden könne ${ }^{54}$. Rigby erwarb sich rasch einen internationalen Ruf. Die guten Resultate seiner Interventionen mochten auch auf seiner Beobachtung der Whiteschen Prinzipien beruhen: Rigby war mit White befreundet; ihm hatte er das Manuskript seines Essay zur Kritik unterbreitet, bevor er es 1775 veröffentlichte ${ }^{55}$.

Gewisse Geburtshelfer werteten also ihre Eingriffe numerisch aus. So wurden etwa die Ergebnisse der 1777 von Jean-René Sigault in einem Fall beschriebenen sectio symphysis pubis allgemein auf Zahlenbasis mit denjenigen des Kaiserschnitts verglichen ${ }^{56}$. Allerdings mußten sie bei der Seltenheit der Fälle einzeln aus der Literatur zusammengesucht werden. Exemplarisch für jene Zeit war Merrimans Vergleich der Ergebnisse dieser beiden Operationen aus der englischen Literatur mit denjenigen der vorzeitigen Einleitung der Geburt aus seiner eigenen Praxis beim Vorliegen eines Ovarialtumors oder einer Beckendeformität. Wesentlich war dabei die Erkenntnis, daß aus der Literatur gesammelte, häufig günstige Einzelfälle nicht ein gleich vollständiges Bild ergaben wie die Berücksichtigung jedes Patienten einer Institution während eines gewissen Zeitraumes. Nachdem Merriman in 33 Fällen alle Mütter und neun Kinder gerettet hatte, die Sterblichkeit der Mütter bei Kaiserschnitt und der Sigaultschen Operation aber über $50 \% \operatorname{lag}^{57}$, konnten ihm die chirurgischen Resultate höchstens noch als «Mahnung» dienen «gegenüber einer unüberlegten, überschnellen Anwendung von Behandlungsmethoden, die weder durch korrekte Überlegungen gerechtfertigt sind noch die Probe der Erfahrung bestanden haben».$^{58}$ In einer Erörterung seiner Statistiken zitierte er 1812 denn auch 
mißbilligend drei französische Autoren, die die künstliche Geburtseinleitung, zugegebenermaßen ohne eigene Erfahrung, ablehnten ${ }^{58}$. Waren religiöse oder gar politische Gründe mit dafür verantwortlich, daß dieses englische Verfahren bald in ganz Europa aufgenommen wurde, in Frankreich jedoch erst nach 1831 Anklang fand ${ }^{59,60}$ ?

IV

Die vorliegende Studie zeigt das Bemühen einer Reihe englischer Geburtshelfer zwischen 1750 und 1820, wichtige, von ihren Vorgängern auf Schätzungsbasis entschiedene Fragen durch einfache arithmetische Analyse zahlreicher Beobachtungen einer sicheren Lösung zuzuführen. Dienten zuerst allgemeine Sterbetafeln als Argumentationsgrundlage für und wider den Zusammenhang zwischen Klima und Krankheit, so wurden sie bald zum Beweis des Wertes von Präventivmaßnahmen herangezogen. Statistiken aus Gebärspitälern lieferten eine unmittelbare Grundlage dafür, und schließlich auch für die Indikation und die Resultate therapeutischen Eingreifens. Dieses Beispiel illustriert den Übergang der statistischen Methode aus dem Bereich der Prävention in denjenigen der klinisch-kurativen Medizin. Es zeigt eine wichtige Wurzel der klinischen Statistik. Damit eingefleischte Gewohnheiten aufgegeben würden, bedurfte es damals wie heute starker Argumente. Dazu waren im Zeitalter der Aufklärung Zahlen willkommen. Daß die Geburtshelfer diese offenbar auch in ihrem Bestreben nach Verdrängung der Hebammen und Anerkennung durch die Kollegen benutzten ${ }^{61}$, verdient ebenfalls Beachtung. Mutatis mutandis gilt dies auch für viele der eingangs erwähnten Spitalärzte und -chirurgen ${ }^{62}$. Es zeigt, daß die oft als mühsam empfundene statistische Auswertung nicht nur mit idealistischer, sondern zuweilen auch mit ganz handgreiflicher Zielsetzung erfolgen konnte ${ }^{63}$.

Mit der Eröffnung von Gebärabteilungen überall in Europa im späten 18. Jahrhundert ${ }^{64}$ finden sich etliche beschreibende Statistiken der Inzidenz von Präsentationen und Mißbildungen, der Geburtsgewichte und Geschlechtsverteilung der Kinder ${ }^{65}$. Sie widerspiegeln ein wachsendes Interesse jener Zeit an Bevölkerungsstatistik ${ }^{66}$. Die Daten dazu waren recht objektiv zusammenzustellen. War etwa die Frage der Schwangerschaftsdauer schon vorher numerisch angegangen worden ${ }^{67}$, so scheinen indessen analysierende klinisch-statistische Berichte über Geburtskomplikationen 
und therapeutische Fragen anfänglich ein britisches Unterfangen gewesen zu sein. Bezeichnenderweise stellte Merriman den britischen Daten die ihm zugänglichen, weniger detaillierten tabellarischen Angaben aus dem Ausland zur Seite ${ }^{68}$. In keinem dieser Berichte, außer jenem aus Mailand von 1811, finden sich indessen sowohl Morbiditäts- als auch Mortalitätsangaben für die verschiedenen Komplikationen und Eingriffe ${ }^{69}$.

$\mathrm{Da} ß$ die Ergebnisse der britischen Geburtshelfer neben zeitlosen Tatsachen viel Zeitgebundenes enthalten, schmälert ihr Verdienst keineswegs, denn die empfohlene arithmetische Auswertung eigener Beobachtungen war zukunftsträchtiger als gleichzeitige Versuche der Quantifizierung rationaler Systeme in der Suche nach «certainty in medicine». ${ }^{70}$ Die Suche zeigt andererseits, daß den führenden britischen Geburtshelfern die Therapie ein ernstes Anliegen war. Mit ihrer Methode reihen sie sich unter die stattliche Zahl der anderen britischen «arithmetischen Observationisten» ein, die damals in Medizin und Chirurgie gleiche Ziele verfolgten ${ }^{71}$. 


\section{Bibliographie und Anmerkungen}

${ }^{1}$ Tröhler, U., Towards clinical investigation on a numerical basis: James Lind at Haslar Hospital 1758-1783. Proc. XXVII. Int. Congr. Hist. Med. Barcelona 1980 (noch nicht erschienen).

2 Tröhler, U., Britische Spitäler und Polikliniken als Heil- und Forschungsstätten 1720-1820. Gesnerus 38 (1981), Heft 3/4 (noch nicht erschienen).

${ }^{3}$ Tröhler, U., Quantification in British medicine and surgery 1750-1830, with special reference to its introduction into therapeutics, London, University College, 1978.

${ }^{4}$ Spencer, H. R., The history of British midwifery from 1650 to 1800, London 1927, repr. New York 1978, S.91, 179-182.

${ }^{5}$ Smellie und White veröffentlichten ausführliche Sammlungen von Einzelfällen, s. Smellie, Anm. 19, bzw. White, C., Cases in surgery, London 1770, und Anm. 27.

${ }^{6}$ King, L. S., The medical world of the eighteenth century, Huntingdon, N. Y., 1971, S. 123-155; Wilson, L.G., Fevers and science in early nineteenth century medicine. J. Hist. Med. 33 (1978) 386-407.

7 Peckham, C.H., A brief history of puerperal infection. Bull. Hist. Med. 3 (1935) 187-212; Cutter, I.S., und H.R.Viets, A short history of midwifery, Philadelphia/London 1964, S. 99-136.

8 ibid.

${ }^{9}$ Woodward, J. H., The British voluntary hospital movement - success or disaster? Ann. Cis. Hist. soc. 4 (1973) 233-254; derselbe, To do the sick no harm. A study of the British voluntary hospital system to 1875, London 1974.

10 Tröhler, op. cit., Anm. 3, S. 151.

${ }^{11}$ Spencer, op. cit., Anm.4, S.179-181.

12 Malouin, M., Histoire des maladies épidémiques de 1746. Observées à Paris en même temps que les différentes températures de l'air. Histoire de l'Académie Royale des Sciences (Paris), Année 1746, S. 151-174.

${ }^{13}$ Cutter, op. cit., Anm. 7, S. 99.

14 Dieser Bericht wurde noch bis anfangs des 19. Jahrhunderts von englischen Autoren zitiert; s. Spencer, op. cit., Anm.4, S.95, 97.

15 ibid., S. 73.

16 ibid., S. $155 \mathrm{f}$.

${ }^{17}$ Leake, J., Practical observations on the childbed fever, London 1772, abgedruckt in: Churchill, F. (ed.), Essays on the puerperal fever and other diseases selected from the writings of British authors previous to the close of the eighteenth century, London, Sydenham Society, 1849, S.117-203; S. 125.

18 ibid., S. 6f., 125, 169, 474. Von Mai 1770 bis September 1772 verlor er von 305 Patienten nur zwei, wie auch die Sterblichkeit an Kindbettfieber in London allgemein vor und nach der Epidemiezeit klein war (1768/69: 185; 1770/71: 172; während der Epidemie 1769/70 aber: 270).

${ }^{19}$ Drei Fälle werden erst im zweiten, postum erschienenen Band der Smellieschen Fallsammlung mitgeteilt. Es handelt sich um die Epikrise dreier im ersten Band beschriebener artifizieller Geburtsbeendigungen durch Kollegen; s. Smellie. W., Observations sur les accouchemens ou suite de la théorie et pratique de cet art, tome III, Paris 1765, S. 512-514. 
${ }^{20}$ Leake, op. cit., Anm.17, S.126. Die Todesursachen wurden von unqualifizierten sog. «death-searchers» angegeben. Die Verarbeitung durch ebensolche «clerks» kannte keine Aufteilung nach Geschlecht und Altersgruppen. Für Beispiele von kritischen Ärzten s. Tröhler, op. cit., Anm. 3, S. 48 f.; 107, 454, und Phil. Trans. Roy. Soc.47 (1752) 333-340.

${ }^{21}$ Greenwood, M., Some British pioneers of social medicine, London/Oxford/Toronto 1948, S.27; Shryock, R.H., The history of quantification in medical science, Isis 52 (1961) 215-237.

${ }^{22}$ Rhodes, P., Doctor John Leake's Hospital, London 1977.

${ }^{23}$ Rolleston, (Sir) H. D., The two Heberdens. Ann. Med. Hist. 5 (1933) 409-427.

${ }^{24}$ Tröhler, op. cit., Anm. 3, S. 133.

25 ibid., S. 50-54.

${ }^{26}$ Spencer, op. cit., Anm.4, S. 112 f., 164.

${ }^{27}$ Vor dem Umzug waren von 403 Patienten in drei Jahren 22 gestorben ( 1 in 18 1/3), nachher von 6459 in sechs Jahren 263 ( 1 in 241/2); s. White, C., A treatise on the management of pregnant and lying-in women, London 1773, in: Churchill, op. cit., Anm. 17, S. 234.

28 Tröhler, op. cit., Anm. 3, S. 152.

${ }^{29}$ White, op. cit., Anm. 27, S. 241-243, 252.

${ }^{30}$ Ich studierte die Arbeiten von Thomas Kirkland (1774) und William Butter (1775) (beide ohne numerische Angaben); Robert Bland (1782), Joseph Clarke (1790), John Clarke (1793) (mit numerischen Angaben), s. Tröhler, op. cit., Anm. 3, S. 153.

31 ibid., S. 287-295; Tröhler, op. cit., Anm. 1, 2; White, op. cit., Anm. 27, S. 254.

${ }^{32}$ Gebärende wurden in der Manchester Infirmary nicht angenommen. Eine separate Gebärklinik wurde dort erst 1790 auf Whites Betreiben eröffnet; s. Cullingworth, C.J., Charles White, F.R.S., a great provincial surgeon and obstetrician of the eighteenth century, London 1904.

${ }^{33}$ s. Tröhler, op. cit., Anm. 3, S.409.

${ }^{34}$ Merriman, S., A synopsis of the various kinds of difficult parturition ..., 3. Aufl., London 1820, S. 305.

${ }^{35}$ Bland, R., Midwifery reports of the Westminster General Dispensary; Summary in: Lond. med. J. 3 (1782) 114-121.

Das Westminster Dispensary war eine vom Gründer John Millar (1733-1805) bewußt auf «arithmetische», klinisch-therapeutische Forschung ausgerichtete Poliklinik; s. Tröhler, op. cit., Anm. 3, S. 110-112.

${ }^{36}$ Clarke, J., Observations on some causes of the excess of the mortality of males above that of females. Phil.Trans.Roy.Soc. 76 (1786), Part II, S.349-364. Später auch abgedruckt in: Lond.med.J.9 (1788) 179-200. - Wie Bland präzisierte auch Clarke anderswo: «Having suppressed nothing, my errors may possibly prove instructive»; s. Clarke, J., Abstract of a registry kept for some years in the Lying-in Hospital of Dublin. Trans. Ass. Fellows Lic. King's Queen's Coll. Phys. Irel.1 (1817) 367-402.

37 Bland, op. cit., Anm. 35.

38 Tröhler, op. cit., Anm. 3, S. 153.

${ }^{39}$ Clarke, Abstract ..., op. cit., Anm. 36, S. 370 f., 399.

${ }^{40}$ Peckham, op. cit., Anm. 7; Cutter und Viets, op. cit., Anm.7, S.109-111. Garrison und Morton's Medical Bibliography, No.6272. - Diese Beweisführung beeindruckte Denman dergestalt, daß er 1801 seine früher vertretene Klima-Ätiologie aufgab - nicht aber die antiphlogistische Therapie; s. Peckham, op. cit. 
${ }^{41}$ London 1795, in: Churchill, op. cit., Anm.17, S.445-500. Gordon hörte bei Denman und/oder dessen Nachfolger; ibid., Fußnote zu S.443-447.

42 ibid., S. 475.

43 ibid., S. 474. Bei genauer Analyse der Tabellen finden sich indessen mehrere Ungereimtheiten zwischen Text und Zahlen! Vgl. S.461, 475.

44 ibid., S. 481, 486.

45 ibid., S. 454-465.

${ }^{46}$ Niebyl, P.H., The English bloodletting revolution, or modern medicine before 1850 . Bull. Hist. Med.51 (1977) 464-483.

47 z. B. Hey: Vorher: 11 Tote von 14 Fällen, nachher: 2 Tote von 36 Fällen; s. Tröhler, op. cit., Anm. 3, S. $155 \mathrm{f}$.

48 ibid.

49 s. Spencer, op. cit., Anm.4, S.53; Bland, op. cit., Anm. 35; Merriman, op. cit., Anm. 34, S. 304 f. Merriman gab Smellies und Leakes Schätzungen wieder

50 Tröhler, op. cit., Anm. 3, S.157f.

51 ibid.

${ }^{52}$ Marr, J.P., Historical background of the treatment of placenta praevia. Bull. Hist. Med.9 (1941) 258-293.

${ }^{53}$ Rigby, E., An essay on the uterine haemorrhage which precedes the delivery of the full-grown foetus, 6. Aufl., London 1822, S. X-XIII.

${ }^{54}$ Von 42 Patientinnen mit placenta praevia waren 31 vor dem Einsetzen der Blutung entbunden worden (alle Mütter gerettet), 9 nach dem Einsetzen der Blutung (alle gestorben), 2 Spontanaborte. Alle 64 «akzidentellen» Blutungen hatten spontan einen guten Verlauf genommen; ibid., S.262-264. Es handelte sich um die Fälle aus Rigbys Privatpraxis, da Gebärende im Spital keine Aufnahme fanden; s. auch Anm. 32.

55 ibid., Widmung auf Titelseite. Cullingworth, op. cit., Anm. 32, S. 40 f., 48.

${ }^{56}$ s. zum Beispiel Prof. Alexander Hamilton, 1801, zit. bei Spencer, op. cit., Anm.4, S.96; William Osborne, 1783, ibid., S.121. - Für Paris s. Fasbender, H., Geschichte der Geburtshülfe, Jena 1906, S. 866-871.

${ }^{57}$ Kriterium war die Anzahl geretteter Leben: Sectio caesarea: 23 Operationen, 10 von 46 Leben gerettet; Sectio symphysis pubis: 44 Operationen, 35 von 88 Leben gerettet; vorzeitige Geburtseinleitung mittels Blasensprengung im achten Monat: 33 Fälle, 9 Kinder und alle Mütter gerettet; zit. bei Tröhler, op. cit., Anm. 3, S. 158 f.

58 ibid.

59 ibid.

${ }^{60}$ Marr, op. cit., Anm. 52.

${ }^{61}$ Dennison, J., Midwives and medical men, London 1977, S.42-61.

62 Tröhler, op. cit., Anm. 3, S.457-461.

63 ibid., S. 450-457; derselbe, op. cit., Anm. 2.

${ }^{64}$ Fasbender, op. cit., Anm. 56, S. 245-264.

${ }^{65}$ Bekannt waren ihm Zahlen aus Amsterdam (1741-1765), Gent (1785), Paris (J.-L. Baudelocque, 1799-1809), Mailand (P.Assalini, 1810) und Wien (L. J. Boër, 1812); s. Merriman, op. cit., Anm. 34, S. 306-322.

${ }^{66}$ Greenwood, M., Medical statistics from Graunt to Farr, Cambridge Univ. Press, 1948; derselbe, op. cit., Anm.21, S. 27. 
${ }^{67}$ Merriman zitierte dazu Tabellen von Mauriceau (im 17.Jahrhundert) und von J.F.G.Dietz, einem Schüler J.G. Roederers (1727-1763) in Göttingen (1757); s. op. cit., Anm. 34, S. $303 \mathrm{f}$.

68 s. Anm. 65.

${ }^{69}$ Merriman reproduzierte noch eine Anzahl Statistiken hier nicht erwähnter, teils anonymer britischer Autoren; ibid.

${ }^{70}$ Risse, G.B., The quest for certainty in medicine: John Brown's system of medicine in France. Bull. Hist. Med.45 (1971) 1-12; s. auch Tröhler, op. cit., Anm.2.

${ }^{71}$ Tröhler, op. cit., Anm. 2, 3.

\section{Summary}

This paper describes the introduction of statistical methods into British midwifery between 1750 and 1820. At first a number of doctors used the London Bills of Mortality, and then specific data derived from one hospital or one private practice as a basis of argument on the theory and therapy of puerperal fever and on the indication for and the results of delivery by art. These obstetricians fit into a movement amongst British doctors around 1780 promoting the analysis of (mass) observations by simple arithmetic as the sure way to gain certainty in medicine.

Priv.-Doz. Dr. med.et phil. Ulrich Tröhler

Medizinhistorische Bibliothek der Universität

Klingelbergstraße 23

4056 Basel 